\title{
Signal changes of the alar ligament in a healthy population: a dispositional or degenerative consequence?
}

\author{
*Holger Wenz, MD, Hans Ulrich Kerl, MD, Mate Elod Maros, MD, Ralf Wenz, BSc (Hons), \\ Kira Kalvin, MD, Christoph Groden, MD, and Ingo Nölte, MD
}

Department of Neuroradiology, Medical Faculty Mannheim, University of Heidelberg, Mannheim, Germany

OBJECT The alar ligaments (ALs) are vital for stabilizing the craniocervical junction. In terms of morphology, their appearance varies and is visible on MRI. Dark signal of the AL on proton-density (PD)-weighted images is generally considered the norm, but the etiology of frequently observed signal hyperintensities is poorly understood. Using spectral fat suppression, signal hyperintensities can be differentiated into fat- and nonfat-related hyperintensities (NFH). Although signal hyperintensities have no evident association with whiplash-associated disorder, age-related degeneration has often been theorized. Therefore, this study investigates the signal intensities of the ALs on 3.0-T MRI with special reference to age. Expanding thereon, the authors investigated the relationship between signal hyperintensities and patient characteristics, such as height, weight, and sex.

METHODS Sixty-six healthy volunteers were scanned using 3.0-T PD-weighted MRI, including spectral fat suppression of the craniocervical junction. The study population was separated into 2 groups (old vs young) using 2 approaches: dichotomization at the median age (40.0 years) and the calculated threshold ( 28.5 years) using receiver operating characteristics (ROC). The AL was independently characterized with respect to continuity, course, shape, signal intensity, and graduation of homogeneity by 2 experienced neuroradiologists. Signal intensity was differentiated into fat-related hyperintensity and NFH. Univariate and multivariate logistic regression models were employed to investigate the relationship between patient characteristics and signal intensities.

RESULTS Two different AL patterns were observed: inhomogeneous (33.3\%) and homogeneous (66.7\%). The latter pattern was mostly surrounded by a small dark rim (56.8\%). Fat could be identified in $15.9 \%$ of all ALs (21 of 132 patients), and NFH was identified in $17.4 \%$ of all ALs (23 of 132 patients). Here, 28.5 years was the preferred threshold, demonstrating a relatively high sensitivity for dichotomizing the population based on the ROC of NFH. The most relevant factor for having NFH was being older than the calculated threshold (odds ratio [OR] 3.420, $p=0.051$ ). Fat-related hyperintensities occurred significantly more frequently in men than women (OR 0.110 and $p=0.007$ for women; OR 9.075 and $p=0.007$ for men). Height was the second most significant factor: for every 1 -cm increase, the odds of having fat lesions increased by approximately $10 \%$ (OR 1.102; $p=0.017$ ).

CONCLUSIONS This study shows that AL signal hyperintensities are substantially influenced by age, sex, and height in healthy individuals. Regarding fat-related hyperintensities, the most relevant factors proved to be sex and height. The odds of detecting NFH increased almost significantly after a relatively young age (> 28.5 years) and were remarkably more frequent in individuals older than 28.5 years. The authors caution presumptions equating signal alterations with age-related deterioration. Instead, they suggest that dispositional factors such as sex and height are more relevant in the AL constitution. Signal alterations in ALs naturally occur in healthy symptom-free individuals, underscoring the importance of cautiously interpreting such lesions on posttraumatic MRI scans.

http://thejns.org/doi/abs/10.3171/2015.1.SPINE141214

KEY WORDS alar ligaments; craniocervical junction; degeneration; fat-related hyperintensity; magnetic resonance imaging; nonfat-related hyperintensity; cervical

\footnotetext{
ABBREVIATIONS AL = alar ligament; FPR = false-positive rate; NFH = nonfat-related hyperintensity; PD = proton density; PD/T2-TSE0 = PD-weighted images/T2-weighted double-echo turbo spin-echo images; ROC = receiver operating characteristics; SD = standard deviation; T2-SPACE = T2-weighted sampling perfection with application optimized contrasts using different flip angle evolution; TPR = true-positive rate; WAD = whiplash-associated disorder.

SUBMITTED November 30, 2014. ACCEPTED January 27, 2015.

INCLUDE WHEN CITING Published online July 17, 2015; DOI: 10.3171/2015.1.SPINE141214.

DISCLOSURE The authors report no conflict of interest concerning the materials or methods used in this study or the findings specified in this paper.

* Drs. Wenz and Kerl contributed equally to this work.
} 
A LAR ligaments (ALs) are vital for stabilizing the craniocervical junction. ${ }^{6,33}$ They connect the dens of the axis to the occipital condyles and inhibit excessive rotation, lateral flexion, as well as anterior dislocation of the atlas on the axis during flexion. ${ }^{4,8,9,13,22,28}$ Substantial variability in the morphology of the upper cervical ligaments has been shown in various studies using MRI. . $6,20,24,29,32,35^{2}$

Dark signals on proton-density (PD)-weighted images of the ALs are generally considered the norm.${ }^{19}$ However, high signal alterations of the ALs have frequently been reported in patients with (chronic) whiplash-associated disorder (WAD), 1,5,11,26,28,31 and also in uninjured individuals. ${ }^{14,16,34}$ This high signal intensity of the ALs was previously suggested by some authors to be correlated with the clinical symptoms caused by the mechanism of whiplash. ${ }^{17,19}$ However, these findings have been contradicted by several investigators who found no direct correlation

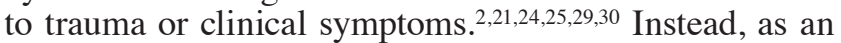
alternative explanation, degeneration has been put forward as the source of these high signal changes. ${ }^{37}$

This hypothesis was put to the test. Are signal changes of the ALs evidence of age-related degeneration? Or are there other influences on AL composition? To test this, 2 neuroradiologists assessed the ALs in healthy volunteers with respect to continuity, course, shape, and graded signal intensity. To the best of our knowledge, this is the first study that evaluates the relationship between physical characteristics such as weight, height, age, and sex, and their respective influences on signal intensities.

\section{Methods \\ Study Cohort}

Sixty-six healthy volunteers who had provided written informed consent were included $53.0 \%$ were female [35 of 66 participants]). Exclusion criteria included neck pain, restricted mobility of the cervical spine, history of trauma to the cervical spine, traumatic brain injury, motor vehicle accident, and medical history of neurological or spinerelated diseases. Furthermore, patients with a history of connective tissue disorders, such as Marfan syndrome and Ehler-Danlos syndrome, were excluded.

To investigate age-related AL deterioration, the study population was separated into 2 groups: old and young. Two different approaches were used: 1) classic dichotomization at the median age (40.0 years); and 2) dichotomization at the optimal threshold (with high sensitivity and specificity) based on the receiver operating characteristic (ROC) for age (28.5 years). This study was performed according to local institutional guidelines and approved by the local ethics committee.

\section{MRI Protocol}

MRI was performed using a 3.0-T magnetic field scanner (Magnetom Trio, Siemens Healthcare). A standard 12-channel head and neck coil was used, and all images were acquired with the participant's head and neck in a neutral position.

The imaging protocol consisted of T2-SPACE (T2weighted sampling perfection with application optimized contrasts using different flip angle evolution) sequences, $\mathrm{PD} / \mathrm{T} 2-\mathrm{TSE}$ (PD-weighted images/T2-weighted doubleecho turbo spin-echo images) with and without fat saturation angulated parallel to the left and right $\mathrm{AL}$, and $1 \mathrm{axial}$ PD/T2-TSE sequence (for details see Table 1).

\section{Image Evaluation}

All viewing and analysis of the acquired data sets were electronically performed on a DICOM workstation using OsiriX Imaging Software (http://www.osirix-viewer.com/ index.html).

Two neuroradiologists, who were blind to all clinical data, independently analyzed each data set. These raters were allowed to freely adjust the window/level settings, but no automatic preprocessing was applied. For optimal evaluation of ligamental continuity, the T2-SPACE sequence was used. The subsequently acquired semicoronal PD-weighted images of the AL were used to evaluate the signal characteristics and determine AL morphology.

With regard to AL morphology, the parameters of continuity, course, and shape from the dens to the occipital condyle were evaluated. These parameters were determined in the axial and coronal reconstructed T2-SPACE (anterolaterally/laterally/dorsolaterally, ascending, strictly horizontally, descending).

Regarding the signal characteristics, homogeneity and signal intensity were determined. AL homogeneity was categorized, on semicoronal PD-weighted images, as homogeneous (Fig. 1) or inhomogeneous (Fig. 2) patterns. The homogeneous pattern included ALs with a hypointense dark rim (Fig. 1C, D). In contrast, the inhomoge-

TABLE 1. MRI parameters for each sequence used in this study

\begin{tabular}{lccc}
\hline \multicolumn{1}{c}{ Parameter } & T2-SPACE $^{*}$ & PD-T2-TSE $\dagger$ & PD-T2-TSE-FS $\neq$ \\
\hline Voxel size $(\mathrm{mm})$ & $0.9 \times 0.9 \times 0.9$ & $0.7 \times 0.5 \times 2.0$ & $0.7 \times 0.5 \times 2.0$ \\
\hline FOV-read (mm) & 280 & 180 & 180 \\
\hline FOV-phase (\%) & 100 & 100 & 100 \\
\hline $\begin{array}{l}\text { Base resolution } \\
\text { (pixel) }\end{array}$ & 230 & 384 & 384 \\
\hline Slices & 52 & 20 & 20 \\
\hline $\begin{array}{l}\text { Slice thickness } \\
\text { (mm) }\end{array}$ & 0.9 & 2 & 2 \\
\hline Averages & 14 & 3 & 3 \\
\hline TR (msec) & 1500 & 4550 & 4550 \\
\hline TE (msec) & 121 & $25 / 116$ & $25 / 116$ \\
\hline Flip angle $\left({ }^{\circ}\right.$ ) & 140 & 150 & 150 \\
\hline Echo-train length & & 8 & 8 \\
\hline Fat saturation & No & No & Yes \\
\hline TA (min) & $05: 53$ & $04: 12$ & $04: 16$ \\
\hline PAT & 3 & 2 & 2 \\
\hline FOV & & & \\
\hline
\end{tabular}

FOV = field of view; PAT = parallel acquisition technique; $T A$ = acquisition time; $\mathrm{TE}=$ echo time; $\mathrm{TR}$ = repetition time.

* SPACE, Sampling Perfection with Application optimized Contrasts using different flip angle Evolution.

† PD-T2-TSE, Proton-Density weighted images/T2-weighted double echo Turbo Spin Echo images.

‡ PD-T2-TSE-FS, Proton-Density weighted images/T2-weighted double echo Turbo Spin Echo images with fat saturation. 


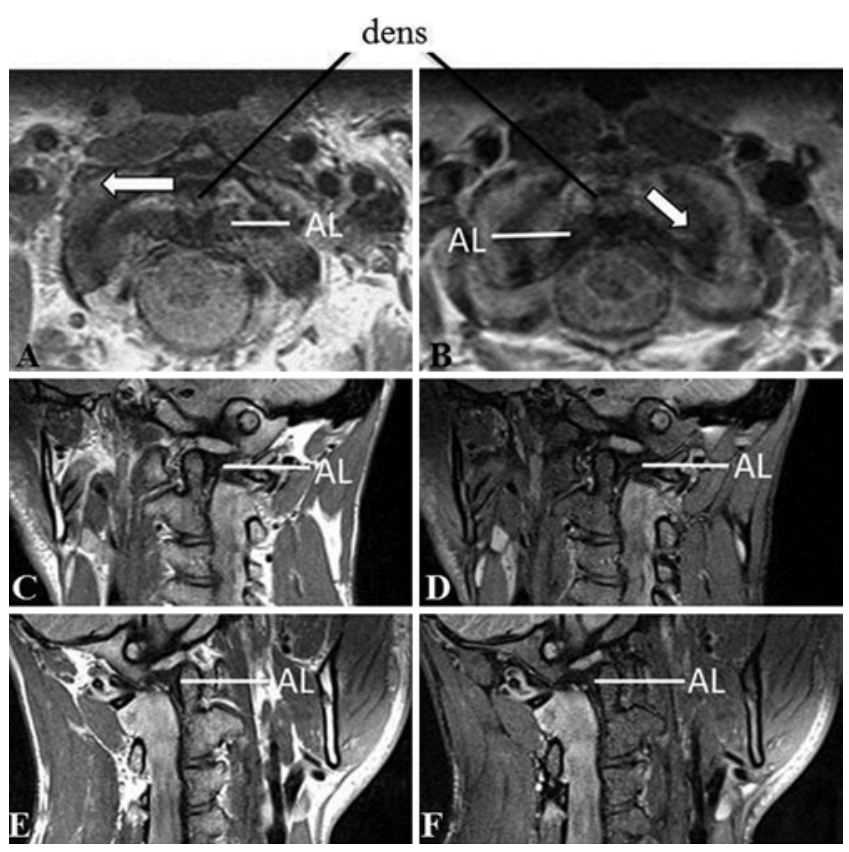

FIG. 1. Courses and signal patterns of the AL on PD-weighted images. White bars reveal the location of the AL. A and B: Axial images showing that the course (arrows) of the AL was either strictly lateral (A) or dorsolateral (B). C and D: Oblique images parallel to the course of the left $A L$, which is laterally ascending, and spectral fat suppression (D). Note the homogeneous signal pattern with the clear dark rim of the $\mathrm{AL}$ in both $C$ and $D$. $E$ and F: Oblique images parallel to the course of the right $\mathrm{AL}$, which is strictly horizontal, and fat suppression $(\mathrm{F})$. Note the homogeneous signal pattern of the $A L$ in both.

neous pattern was further graded based on the extent of inhomogeneity according to Kråkenes et al. ${ }^{16}$ (Table 2). Importantly, signal intensity was further differentiated into either fat-related hyperintensity or nonfat-related hyperintensity (NFH). This differentiation was achieved using spectral fat suppression.

\section{Statistical Analysis}

The statistical analyses were descriptive and performed using SPSS software (Statistical Package for the Social Sciences, version 19.0; IBM Corporation). The distributions of age, height, and weight were tested against the normal distributions using the Shapiro-Wilk test and Q-Q plots. We defined the following binary outcome parameters: the presence of 1) high signal intensity; 2) fat signal; and 3) NFH, either in the left or right AL.

ROC was plotted to measure sensitivity and the falsepositive rate at the possible thresholds for the clinical features of age, height, and weight. The best-performing age threshold was used to dichotomize the data set in further analyses.

Univariate logistic regression was used to assess the relationship between the patient characteristics (age, weight, height, and sex) and the 3 aforementioned outcome parameters. We also used multivariate-adjusted models to isolate the effects of certain clinical features. To evaluate signal differences between age groups, the chi-square or Fisher exact test was used. We applied Pearson's correlation to assess the relationship between weight and height. To
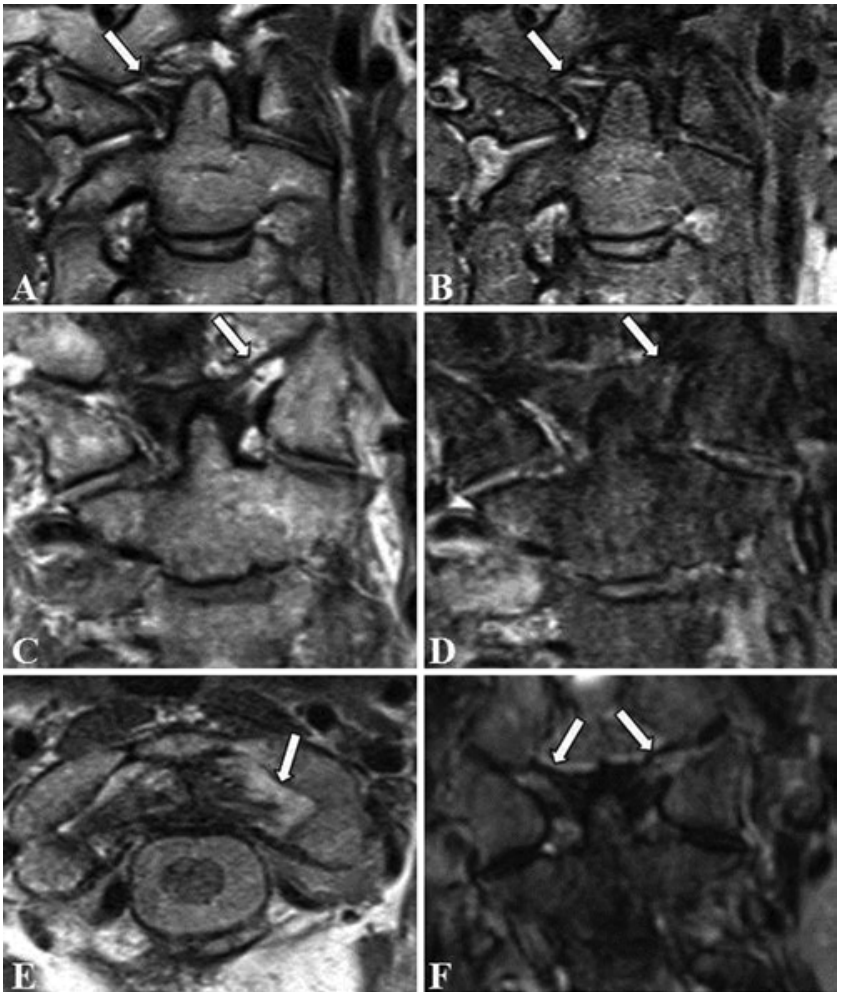

FIG. 2. Hyperintensity types ([A and $B]$ and fat $[C-F])$ in $P D$-weighted images. $A$ and $B$ : Oblique images of the right $A L$ obtained in a 20 -yearold male, including spectral fat suppression (B). There are linear hyperintensities (arrows) along the course of the ligament (both $A$ and $B$ ), which cannot be suppressed by spectral fat suppression (B) and hence are NFH. The left ligament was homogeneously dark. C-F: Fat signal of left $\mathrm{AL}$ insertion at the occipital condyle in a 52-year-old male. Panels $\mathrm{C}$ (oblique) and $\mathrm{E}$ (axial) show bright signal hyperintensities, while $\mathrm{D}$ (oblique) and $F$ (axial) illustrate the corresponding decreased signal after spectral fat suppression and, hence, are fat-related hyperintensities. Asymmetrical signal of both ALs (multiplanar coronal reconstruction of a SPACE data set) is shown in panel $F$.

compare the age, weight, and height distributions between men and women, the Wilcoxon-Mann-Whitney U-test or unpaired t-test was applied, respectively. In this study, $\mathrm{p}$ values $<0.05$ were considered statistically significant.

\section{Results}

\section{Study Cohort}

The ages of the volunteers ranged between 18 and 65 years, with a median of 40.0 years (lower-upper quartile 25.75-52.25 years) and peaks around 20-25 and 55-60 years. Both height (range 149-198 cm; mean $172.9 \pm 9.3$ cm [SD]) and weight (range 51-105 kg; mean $74 \pm 12.5 \mathrm{~kg}$ ) approximately followed a normal distribution.

\section{TABLE 2. Grading criteria for the extent of inhomogeneity of ALs}

\begin{tabular}{cl}
\hline Grade & Criteria \\
\hline 0 & Low signal intensity throughout the entire cross-section \\
\hline 1 & High signal intensity in one-third or less of cross-section \\
\hline 2 & High signal intensity in one-third to two-thirds of cross-section \\
\hline 3 & High signal intensity in two-thirds or more of cross-section \\
\hline
\end{tabular}




\section{Continuity, Course, and Shape of ALs}

All 132 ALs (66 volunteers; 1 AL on each side) showed a continuous course on the T2-SPACE sequences. In 1 case, the PD-weighted sequences were equivocal concerning $1 \mathrm{AL}$. In this case, continuity was confirmed with the T2-SPACE sequences. Symmetry, in terms of shape and size, was present in $91 \%$ of participants (60 of 66); asymmetrical AL was found in 9\% of participants (6 of 66).

The course of the AL in the coronal plane was laterally ascending in $62.1 \%$ (41 of 66) (Fig. 1C and D) and horizontally oriented in $37.9 \%$ of participants (25 of 66) (Fig. 1E and F). No laterally descending course was observed. The course of the AL in the axial plane was strictly laterally oriented in $40.9 \%$ (27 of 66) (Fig. 1A) and dorsolaterally in $59.1 \%$ of participants (39 of 66) (Fig. 1B). Anterolateral orientation was not detected.

\section{Patterns of Signal Characteristics of the ALs}

Concerning the signal characteristics of ALs, 2 different patterns were observed: homogeneous (with or without a dark rim) and inhomogeneous.

A homogeneous signal pattern for the AL presented in $66.7 \%$ (88 of 132) of participants. This pattern, however, was subdivided into the completely homogeneous pattern (Fig. 1E and F) and the homogeneous pattern with a small dark rim (Fig. 1C and D). Only a minority of ALs were completely homogeneous (9.9\%; 13 of 132 participants; 7 left- and 6 right-sided AL). In $56.8 \%$ of all homogeneous cases (75 of 132 participants; 35 left- and 40 right-sided AL), an intermediate to bright signal surrounded by a small dark rim of various thicknesses was seen.

The inhomogeneous signal pattern of the ALs (Fig. 2) was observed in one-third of all participants (44 of 132 participants; 24 left- and 20 right-sided ALs). In the vast majority of these participants $(93.18 \%$; 41 of 44 participants; 21 left-sided and 20 right-sided ALs), the extent of inhomogeneity was less than one-third of the AL crosssection. An inhomogeneity of more than one-third was rare $(6.81 \% ; 3$ of 44 participants; 3 left- and 0 right-sided $\mathrm{AL})$. Moreover, an extended inhomogeneity of more than two-thirds was not found.

Fat could be identified in 21 ALs (9 right- and 12 leftsided ALs) using spectral fat suppression, reflecting $15.9 \%$ (21 of 132) of all ligaments (Fig. 2C and D). All of these 21 cases belonged to the inhomogeneous AL subgroup, representing $47.7 \%$ of participants (21 of 44). Likewise, $\mathrm{NFH}$ could be detected in $17.4 \%$ of participants (23 of 132) (Fig. $2 \mathrm{~A}$ and $\mathrm{B})$.

\section{Receiver Operating Characteristics}

ROC analysis was used to identify an ideal age threshold. The ROC of the age for showing NFH demonstrated a weak tendency, with an area under the curve of 0.573 (95\% CI 0.434-0.713; $\mathrm{p}=0.334)$. A suitable age threshold was set at 28.5 years (23 of 66 participants were < 28.5 years; 43 of 66 participants were $\geq 28.5$ years) in order to achieve a relatively high sensitivity of $81.8 \%$ (i.e., true-positive rate [TPR]) with a corresponding acceptable specificity of $43.2 \%$ (i.e., relatively low false-positive rate [FPR] of 56.8\%). In comparison with the ROC approach, classic dichotomization at the median age (40.0 years) resulted in a sensitivity (i.e., TPR) of $59.1 \%$ and a specificity (FPR) of $45.5 \%$.

\section{Relationship Between Signal Hyperintensity Types and Patient Characteristics \\ General Signal Hyperintensity}

Men had a tendency $(p=0.137)$ for $111 \%$ increased odds of having nonspecific hyperintensity in AL (OR 2.111 [95\% CI 0.022-0.549]) in comparison with women. Bodyweight showed a similar trend; with every 1-kg increase the odds of having an AL-hyperintensity increased by approximately $2.9 \%$ (OR 1.029 [95\% CI 0.988-1.072], $\mathrm{p}=0.173)$.

\section{Nonfat-Related Hyperintensity}

In the univariate logistic regression, age showed only a weak tendency with NFH(OR 1.020 [95\% CI0.982-1.059], $\mathrm{p}=0.315$ ). However, when comparing the ROC values for the age thresholds, the older subgroup ( $\geq 28.5$ years) had an almost statistically significant $(\mathrm{p}=0.051)$ and clinically highly relevant ( $240 \%$ increased odds) (OR 3.420 [95\% CI 0.993-11.780]) of having NFH in their ALs. Crosstabulation showed similar results $(\mathrm{p}=0.057)$. In contrast, classic dichotomization at the median age resulted only in a weak tendency toward an increased odds of having NFH (OR 1.733 [95\% CI 0.615-4.887], $\mathrm{p}=0.298$ ).

In the multivariate logistic regression model adjusted for sex, height, and weight, patients $\geq 28.5$ years had a clinically relevant tendency for an increased chance of having NFH (OR 2.849 [95\% CI 0.742-10.948], p = 0.127). The other markers showed no relevant trend or interaction.

\section{Fat-Related Hyperintensity}

The univariate logistic regression analyses were used to elucidate the occurrence of fat-related hyperintensities (i.e., fat lesions). Men and women presented opposite tendencies in their odd ratios for having fat lesions: women had a statistically and highly significant decreased 0.110 OR for having fat lesions (95\% CI $0.022-0.549$, p $=0.007)$. In contrast, men had a statistically highly significant increased 9.075 OR for having fat lesions $(95 \%$ CI 1.822-45.208, $\mathrm{p}=0.007)$. Moreover, in the multivariate model adjusted for age, height, and weight, men had an almost significant trend for an approximately 590\% increased odds for having fat signals in ALs (OR 6.910 [95\% CI 0.855-55.861]; $\mathrm{df}=1 ; \mathrm{p}=0.070)$. No other factor showed a relevant association.

Height had an impact on the occurrence of fat lesions; for every $1-\mathrm{cm}$ increase in height, the odds of having fat lesions increased statistically significantly by approximately $10 \%$ (OR 1.102 [95\% CI 1.018-1.192], p = 0.017). In contrast, weight presented an insignificant tendency (OR 1.037 [95\% CI 0.986-1.091], $\mathrm{p}=0.155$ ). The data proved the well-known correlation between height and weight at a statistically highly significant level (Pearson $r=0.582$; $\mathrm{n}=65 ; \mathrm{p}<0.01)$. Furthermore, it is noteworthy that men were significantly taller $(\mathrm{p}<0.001)$ and weighed $(\mathrm{p}<$ 0.001 , t-test) more than women without any relevant age differences ( $\mathrm{p}=0.634$, Mann-Whitney U-test) in the study 
cohort. There was no significant interaction between sex and weight or height; similarly, there was no significant interaction between age and the aforementioned factors, or between height and weight.

\section{Discussion}

Substantial morphological variability of the upper cervical ligaments has been shown in various MRI studies, including the ALs. ${ }^{10,16,20,24,29,32,35}$ Signal changes in the ALs associated with trauma were initially reported by Volle ${ }^{38,39}$ and Krakenes ${ }^{16,17}$ and their colleagues in patients with chronic WAD. Furthermore, clinical symptoms were reported in patients with WAD and AL signal alterations. ${ }^{16-18,39}$ However, the clinical significance of these signal changes has been disputed since the 1990s: various MRI studies showed that there is a broad range of variations in interindividual signal intensity and morphology in AL, both in patients with WAD ${ }^{36-40}$ and healthy volunteers. ${ }^{23,24,29}$ As a cautionary note, MRI should generally be accompanied in WAD, and especially in suspected upper spinal instability, with multimodal imaging procedures depending on the clinical setting of the patient. Especially for suspected upper spinal injuries and trauma, it is crucial to evaluate the status of the tectorial membrane, in particular, as it is the single most important stabilizing structure at the craniocervical junction. ${ }^{12,27}$

While direct associations between trauma, clinical symptoms, and signal alterations of the AL were ruled out in recent years, ${ }^{2,21,24,25,29,30}$ age-related degenerative changes have been assumed as an alternative explanation for signal changes ${ }^{37}$ However, to date, concrete evidence that substantiates these claims is lacking. The goal of this cross-sectional study was to explore if signal intensity and signal changes are influenced by age. Accordingly, we assessed the morphological variations and signal intensity of the ALs in an asymptomatic cohort of 2 groups of different ages (young vs old). Moreover, we statistically evaluated the relationship between other patient characteristics, such as weight, height, and sex, and their respective signal intensities.

Morphologically, our results are consistent with prior in vitro $^{8}$ and in vivo $^{20,24,29}$ studies regarding $\mathrm{AL}$ orientation. On coronal images, $62.1 \%$ were laterally ascending and $37.9 \%$ were horizontally orientated. In accordance with our results, Lummel et al. ${ }^{24}$ described a laterally ascending orientation in $58.5 \%$ and a horizontal orientation in $40.5 \%$ of patients.

Regarding the features of the signal patterns, we observed 2 different kinds. On one hand, a homogeneous intermediate to bright signal pattern, usually surrounded by a small dark rim of various thicknesses, was noted in $66.7 \%$ of all AL. On the other hand, we observed an inhomogeneous signal pattern in one-third of patients.

The signal alterations' origin may have different causes. First, it could be due to the misinterpretation of the partial volume effects from the surrounding fatty tissues. Second, it may be due to scars from severe craniocervical injuries in patients with high-energy traumata. This type of population was excluded from our study. Third, it could be caused by dispersed interstitial fat within the AL, which was shown by Koch in 2008 in her autopsy study.${ }^{15}$ Lastly, AL signal alterations may presumably be due to age-related degeneration. Investigating this presumption, the hyperintensities were either categorized as fat-related hypersensitivity (interstitial fat in ALs) or NFH, depending on the signal in the fat-suppressed PD-weighted sequences.

Using the fat suppression sequences, we found NFH in 23 of 132 ALs. Our analysis showed that the older age group ( $\geq 28.5$ years) had an almost significant and relevant increased odds by approximately $240 \%$ for having NFH. Setting the age threshold at a relatively young age of 28.5 years, we emphasized higher sensitivity at the price of slightly lower specificity for detecting NFH lesions. Nonetheless, the specificity was almost as good as the general approach using the median age. No other factor seemed to significantly influence the occurrence of NFH in our patients.

Regarding fat-related hyperintensities, we found that age did not play a significant factor, neither did classical dichotomization at the median age of 40 years nor for the threshold age of 28.5 years. Surprisingly, the factors most relevant to having fat-related hyperintensity appeared to be sex followed by height. Female patients had a significantly decreased odds ratio of having fat lesions, while men had statistically highly increased odds of having fat lesions. For men, the odds of having fat lesions in the multivariate model were increased by approximately $590 \%$, and in the univariate model the odds were increased by approximately $800 \%$. In contrast, females had a $90 \%$ decreased odds of having fat lesions. Moreover, counterintuitively, weight did not significantly impact the odds of having fat-related hyperintensity, while height did. For every $1-\mathrm{cm}$ increase in height, the odds of having fat lesions significantly increased by approximately $10 \%$. However, it is noteworthy that, as expected, men were significantly taller and weighed more than female participants, which could bias the perceived frequency of the signal alterations.

To recapitulate, the most relevant factor for having NFH was older age, as calculated by the ROC analysis. In contrast, for fat-related hyperintensities, sex and height seemed to influence fat lesion occurrence the most, with men being more likely and females being less likely to demonstrate fat lesions.

Because we are the first to show that the aforementioned factors affect AL constitution, one might argue that a limitation of our study is that the findings might be somewhat incidental due to the moderate population size ( $\mathrm{n}=$ 66). However, comparison with other studies shows that our findings are indeed viable. Using fat suppression, Dullerud et al. ${ }^{7}$ was among the first to differentiate between $\mathrm{NFH}$ and intraligamentous fat of the AL. In Dullerud and colleagues' control population of healthy volunteers $(n=$ 27), the observed frequency of signal alterations of the AL without fat suppression was $54 \%$; with fat-suppression (i.e., NFH) the frequency was $37 \%$. Therefore, in $17 \%$ of the control population, fat was detectable within the AL. These results are very similar to our results that about $16 \%$ of ALs contain fat. In our study, NFH was detectable in 52.3\% of all ligaments with bright signal alterations. Therefore, indeed, our results appear to be reproducible.

Another possible limitation of our study might be the 
impediment of evaluating small structures due to partial volume effects. However, an autopsy series ${ }^{3}$ of relatively small AL reported the dimensions to be about $8.8 \pm 2.6$ $\mathrm{mm}$ in length and $7.3 \pm 1.3 \mathrm{~mm}$ in diameter. Therefore, applying 2-mm-thick PD-weighted images without a gap is reliable and well established. ${ }^{20,23,24}$ Furthermore, one could further argue that higher MRI field strength could improve AL delineation, increase AL signal intensities, and, thus, adulterate imaging evaluations. This, however, was ruled out before. ${ }^{23}$

Lastly, in the clinical setting of assessing craniocervical junction integrity and the resulting decision-making management, it is crucial to rely on various examinations in context. This encompasses multimodal imaging procedures of the bony and soft tissue, according to the patient's clinical picture and anamnesis, and also entails not focusing on a single ligamentous structure but evaluating the craniocervical junction as a whole.

\section{Conclusions}

We show that the ALs have signal variability, which is likely influenced by age, sex, and height. The impact on fat-related hyperintensities by sex and height is significant and highly relevant, and therefore crucial to consider when evaluating the patient's clinical status. Moreover, the impact of age on NFH is highly relevant and edges very closely to the significance level. The question of whether AL signal alterations are evidence for age-related degeneration must be answered in 2 ways. First, regarding fat-related hyperintensities, these signal changes are not evidence of age-related deterioration. Second, regarding NFH, these signal changes show a trend toward an increased likelihood of being more prevalent in older individuals.

Therefore, we caution against presumptions equating signal alterations with age-related deterioration for fatrelated hyperintensity, and instead suggest that dispositional factors such as sex and height are more important. In contrast, NFH seem to be an age-related occurrence. In general, signal alterations in AL seem to naturally occur in healthy symptom-free individuals, underscoring the importance of the cautious interpretation of such lesions on posttraumatic MRI scans.

\section{References}

1. Adams VI: Neck injuries: III. Ligamentous injuries of the craniocervical articulation without occipito-atlantal or atlanto-axial facet dislocation. A pathologic study of 21 traffic fatalities. J Forensic Sci 38:1097-1104, 1993

2. Bitterling H, Stäbler A, Brückmann H: [Mystery of alar ligament rupture: value of MRI in whiplash injuries-biomechanical, anatomical and clinical studies.] Rofo 179:11271136, 2007 (Ger)

3. Cattrysse E, Barbero M, Kool P, Gagey O, Clarys JP, Van Roy P: 3D morphometry of the transverse and alar ligaments in the occipito-atlanto-axial complex: an in vitro analysis. Clin Anat 20:892-898, 2007

4. Crisco JJ III, Panjabi MM, Dvorak J: A model of the alar ligaments of the upper cervical spine in axial rotation. $\mathbf{J}$ Biomech 24:607-614, 1991

5. Dickman CA, Greene KA, Sonntag VK: Injuries involving the transverse atlantal ligament: classification and treatment guidelines based upon experience with 39 injuries. Neurosurgery 38:44-50, 1996
6. Dickman CA, Mamourian A, Sonntag VK, Drayer BP: Magnetic resonance imaging of the transverse atlantal ligament for the evaluation of atlantoaxial instability. J Neurosurg 75:221-227, 1991

7. Dullerud R, Gjertsen O, Server A: Magnetic resonance imaging of ligaments and membranes in the craniocervical junction in whiplash-associated injury and in healthy control subjects. Acta Radiol 51:207-212, 2010

8. Dvorak J, Panjabi MM: Functional anatomy of the alar ligaments. Spine (Phila Pa 1976) 12:183-189, 1987

9. Dvorak J, Schneider E, Saldinger P, Rahn B: Biomechanics of the craniocervical region: the alar and transverse ligaments. J Orthop Res 6:452-461, 1988

10. Elliott J, Pedler A, Kenardy J, Galloway G, Jull G, Sterling M: The temporal development of fatty infiltrates in the neck muscles following whiplash injury: an association with pain and posttraumatic stress. PLoS ONE 6:e21194, 2011

11. Fielding JW, Cochran Gv, Lawsing JF III, Hohl M: Tears of the transverse ligament of the atlas. A clinical and biomechanical study. J Bone Joint Surg Am 56:1683-1691, 1974

12. Harris MB, Duval MJ, Davis JA Jr, Bernini PM: Anatomical and roentgenographic features of atlantooccipital instability. J Spinal Disord 6:5-10, 1993

13. Heller JG, Amrani J, Hutton WC: Transverse ligament failure: a biomechanical study. J Spinal Disord 6:162-165, 1993

14. Kaale BR, Krakenes J, Albrektsen G, Wester K: Whiplashassociated disorders impairment rating: neck disability index score according to severity of MRI findings of ligaments and membranes in the upper cervical spine. J Neurotrauma 22:466-475, 2005

15. Koch S: Magnetresonanztomographische, pathoanatomische und histologische Korrelation von Ligamenta alaria an humanen Präparaten nach gewaltsamem Tod [dissertation]. Munich: Ludwig-Maximilian University, 2008

16. Krakenes J, Kaale BR: Magnetic resonance imaging assessment of craniovertebral ligaments and membranes after whiplash trauma. Spine (Phila Pa 1976) 31:2820-2826, 2006

17. Krakenes J, Kaale BR, Moen G, Nordli H, Gilhus NE, Rorvik J: MRI assessment of the alar ligaments in the late stage of whiplash injury-a study of structural abnormalities and observer agreement. Neuroradiology 44:617-624, 2002

18. Krakenes J, Kaale BR, Moen G, Nordli H, Gilhus NE, Rorvik $\mathrm{J}$ : MRI of the tectorial and posterior atlanto-occipital membranes in the late stage of whiplash injury. Neuroradiology 45:585-591, 2003

19. Krakenes J, Kaale BR, Nordli H, Moen G, Rorvik J, Gilhus NE: MR analysis of the transverse ligament in the late stage of whiplash injury. Acta Radiol 44:637-644, 2003

20. Krakenes J, Kaale BR, Rorvik J, Gilhus NE: MRI assessment of normal ligamentous structures in the craniovertebral junction. Neuroradiology 43:1089-1097, 2001

21. Li Q, Shen H, Li M: Magnetic resonance imaging signal changes of alar and transverse ligaments not correlated with whiplash-associated disorders: a meta-analysis of case-control studies. Eur Spine J 22:14-20, 2013

22. Linnman C, Appel L, Fredrikson M, Gordh T, Söderlund A, Långström B, et al: Elevated [11C]-D-deprenyl uptake in chronic Whiplash Associated Disorder suggests persistent musculoskeletal inflammation. PLoS ONE 6:e19182, 2011

23. Lummel N, Schöpf V, Bitterling H, Zeif C, Kloetzer A, Brückmann $\mathrm{H}$, et al: Effect of magnetic resonance imaging field strength on delineation and signal intensity of alar ligaments in healthy volunteers. Spine (Phila Pa 1976) 37:E1062-E1067, 2012

24. Lummel N, Zeif C, Kloetzer A, Linn J, Brückmann H, Bitterling $\mathrm{H}$ : Variability of morphology and signal intensity of alar ligaments in healthy volunteers using MR imaging. AJNR Am J Neuroradiol 32:125-130, 2011 
25. Myran R, Kvistad KA, Nygaard OP, Andresen H, Folvik M, Zwart JA: Magnetic resonance imaging assessment of the alar ligaments in whiplash injuries: a case-control study. Spine (Phila Pa 1976) 33:2012-2016, 2008

26. Obenauer S, Herold T, Fischer U, Fadjasch G, Koebke J, Grabbe E, et al: [The evaluation of experimentally induced injuries to the upper cervical spine with a digital x-ray technic, computed tomography and magnetic resonance tomography.] Rofo 171:473-479, 1999 (Ger)

27. Oda T, Panjabi MM, Crisco JJ III, Bueff HU, Grob D, Dvorak J: Role of tectorial membrane in the stability of the upper cervical spine. Clin Biomech (Bristol, Avon) 7:201-207, 1992

28. Panjabi M, Dvorak J, Crisco J III, Oda T, Hilibrand A, Grob D: Flexion, extension, and lateral bending of the upper cervical spine in response to alar ligament transections. J Spinal Disord 4:157-167, 1991

29. Pfirrmann CW, Binkert CA, Zanetti M, Boos N, Hodler J: MR morphology of alar ligaments and occipitoatlantoaxial joints: study in 50 asymptomatic subjects. Radiology 218:133-137, 2001

30. Roy S, Hol PK, Laerum LT, Tillung T: Pitfalls of magnetic resonance imaging of alar ligament. Neuroradiology 46:392-398, 2004

31. Saldinger P, Dvorak J, Rahn BA, Perren SM: Histology of the alar and transverse ligaments. Spine (Phila Pa 1976) 15:257-261, 1990

32. Schmidt P, Mayer TE, Drescher R: Delineation of alar ligament morphology: comparison of magnetic resonance imaging at 1.5 and 3 Tesla. Orthopedics 35:e1635-e1639, 2012

33. Tubbs RS, Hallock JD, Radcliff V, Naftel RP, Mortazavi M, Shoja MM, et al: Ligaments of the craniocervical junction. J Neurosurg Spine 14:697-709, 2011

34. Vetti N, Kråkenes J, Ask T, Erdal KA, Torkildsen MD, Rørvik J, et al: Follow-up MR imaging of the alar and transverse ligaments after whiplash injury: a prospective controlled study. AJNR Am J Neuroradiol 32:1836-1841, 2011

35. Vetti N, Kråkenes J, Damsgaard E, Rørvik J, Gilhus NE,
Espeland A: Magnetic resonance imaging of the alar and transverse ligaments in acute whiplash-associated disorders 1 and 2: a cross-sectional controlled study. Spine (Phila Pa 1976) 36:E434-E440, 2011

36. Vetti N, Kråkenes J, Eide GE, Rørvik J, Gilhus NE, Espeland A: Are MRI high-signal changes of alar and transverse ligaments in acute whiplash injury related to outcome? BMC Musculoskelet Disord 11:260, 2010

37. Vetti N, Kråkenes J, Eide GE, Rørvik J, Gilhus NE, Espeland A: MRI of the alar and transverse ligaments in whiplashassociated disorders (WAD) grades 1-2: high-signal changes by age, gender, event and time since trauma. Neuroradiology 51:227-235, 2009

38. Volle E: Functional magnetic resonance imaging - video diagnosis of soft-tissue trauma to the craniocervical joints and ligaments. Int Tinnitus J 6:134-139, 2000

39. Volle E, Montazem A: MRI video diagnosis and surgical therapy of soft tissue trauma to the craniocervical junction. Ear Nose Throat J 80:41-44, 46-48, 2001

40. Wong ST, Ernest K, Fan G, Zovickian J, Pang D: Isolated unilateral rupture of the alar ligament. J Neurosurg Pediatr 13:541-547, 2014

\section{Author Contributions}

Conception and design: H Wenz, Kerl, R Wenz, Nölte. Acquisition of data: H Wenz, R Wenz, Kalvin. Analysis and interpretation of data: $\mathrm{H}$ Wenz, Maros, $\mathrm{R}$ Wenz. Drafting the article: $\mathrm{H}$ Wenz, Maros, R Wenz. Critically revising the article: Kerl, $\mathrm{R}$ Wenz. Reviewed submitted version of manuscript: H Wenz, Maros. Statistical analysis: Maros. Administrative/technical/material support: H Wenz. Study supervision: Kalvin, Groden, Nölte.

\section{Correspondence}

Holger Wenz, Department of Neuroradiology, Medical Faculty Mannheim, University of Heidelberg, Theodor-Kutzer-Ufer 1-3, 68167 Mannheim, Germany. email: holger-wenz@gmx.de. 